\section{Ketamine-related uropathy and cholangiopathy}

\section{Yi-Hsuan Chen ${ }^{1}$, Shian-Shiang Wang ${ }^{2}$ and Sung-Yuan $\mathrm{Hu}^{1,3-5 *}$}

1'Department of Emergency Medicine, Taichung Veterans General Hospital, Taiwan 2Division of Urology, Department of Surgery, Taichung Veterans General Hospital, Taiwan

${ }^{3}$ School of Medicine, Chung Shan Medical University, Taiwan

${ }^{4}$ Institute of Medicine, Chung Shan Medical University, Taiwan

${ }^{5}$ Department of Nursing, College of Health, National Taichung University of Science and Technology, Taichung, Taiwan

\section{Clinical image}

A 23-year-old man had a 2-year history of ketamine abuse and presented intermittent abdominal pain, urinary urgency and dysuria for one year. Two weeks ago, laboratory analysis showed within normal limits. This time, he visited our emergency department due to hematuria and bilateral flank pain. CT scan and MRI revealed bilateral hydronephrosis, hydroureter, irregular thickened wall of urinary bladder, and fusiform common bile duct with distal stenosis (Figure 1A, 1B, 1C). Cystoscopy demonstrated ketamine-associated ulcerative cystitis (KAUC) (Figure 1D). Condition became better after bilateral percutaneous nephrostomy and ceasing ketamine abuse. Figure 2 showed serial renal and liver function profiles.

KAUC was first identified by lower urinary tract symptoms (LUTS) in 2007. Mechanisms of ketamine-related urological and gastrointestinal damages include direct toxic injury, microvascular damage, and autoimmunity triggered by ketamine and its metabolites. Clinicians should be aware of this clinical entity and able to recognize it when patients present unexplained gastrointestinal symptoms and LUTS [1,2].

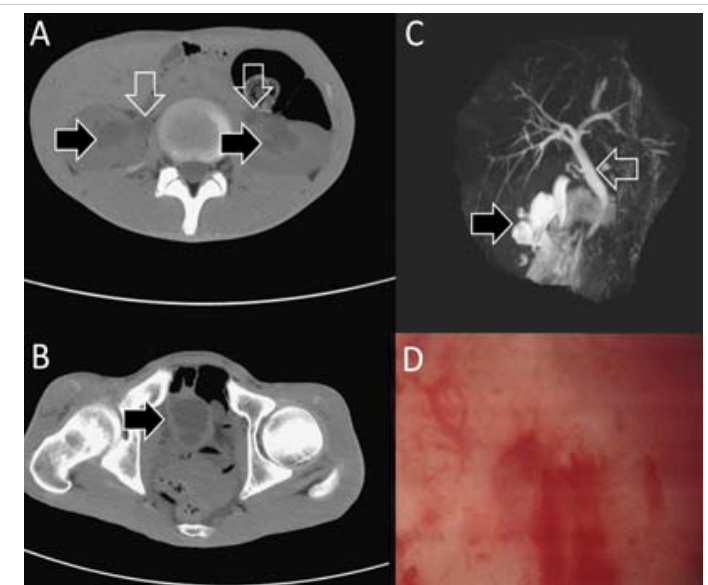

Figure 1: Bilateral hydronephrosis (black arrows in panel A), hydroureter (white arrows in panel A), irregular thickened wall of urinary bladder (black arrow in panel B), fusiform common bile duct with distal stenosis (panel C), and ulcerative cystitis (panel D).

\section{More Information}

*Address for Correspondence: Sung-Yuan Hu, Department of Emergency Medicine, Taichung Veterans General Hospital, 1650 Taiwan Boulevard Sect. 4, Taichung 40705, Taiwan, Tel: +886 4 23592525; ext 3601; Fax: +886 423594065 ;

Email: song9168@pie.com.tw

Submitted: March 15, 2021

Approved: March 17, 2021

Published: March 18, 2021

How to cite this article: Chen $\mathrm{YH}$, Wang SS, Hu SY. Ketamine-related uropathy and cholangiopathy. J Clin Med Exp Images. 2021; 5: 002-002

DOI: 10.29328/journal.jcmei.1001018

Copyright: @ 2021 Chen YH, et al. This is an open access article distributed under the Creative Commons Attribution License, which permits unrestricted use, distribution, and reproduction in any medium, provided the original work is properly cited.

Keywords: Cholangiopathy; Ketamine abuse; Uropathy

\section{(D) Check for updates \\ (๑) OPEN ACCESS}

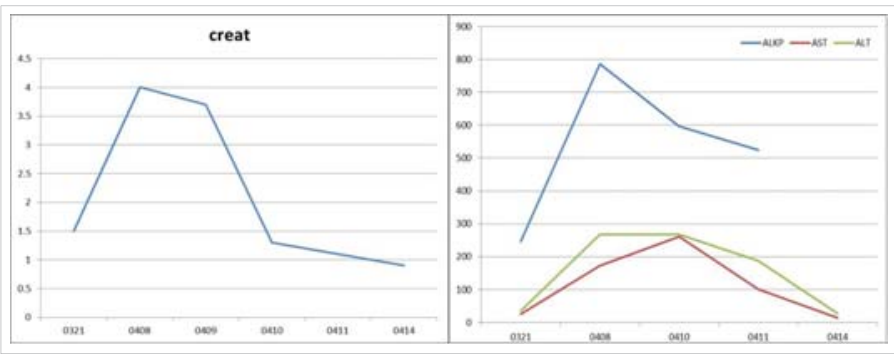

Figure 2: Creatinine (left panel), ALKP, AST, and ALT (right panel) returned to normal limits.

\section{Consent}

This study was approved by the Institutional Review Board of Taichung Veterans General Hospital (No. CE19152A).

This work was supported by grants from the Taichung Veterans General Hospital (Grant numbers: TCVGH-109 7202C).

\section{References}

1. Middela S, Pearce I. Ketamine-induced vesicopathy: a literature review. Int J Clin Pract. 2011; 65: 27-30.

PubMed: https://pubmed.ncbi.nIm.nih.gov/21155941/

2. Yu WL, Cho CC, Lung PF, Hung EHY, Hui JWY, et al. Ketaminerelated cholangiopathy: a retrospective study on clinical and imaging findings. Abdom Imaging. 2014; 39: 1241-1246.

PubMed: https://pubmed.ncbi.nlm.nih.gov/24934474/ 